\title{
Minimally invasive surgical management of symptomatic uterine cysts
}

\author{
Atef M. Darwish • Kamal M. Zahran • \\ Mohammad A. Bedaiwi • Mahmoud S. Zakherah
}

Received: 18 March 2010 /Accepted: 30 June 2010 /Published online: 21 July 2010

(C) Springer-Verlag 2010

\begin{abstract}
This study aims to evaluate the feasibility of a minimally invasive access-integrated protocol for aspiration or drainage of symptomatic uterine cysts. The design of the study is a prospective cohort study. The study setting is a tertiary care referral facility and university hospital. Twenty seven women with objective evidence of uterine cysts diagnosed by ultrasonography. The patients underwent transvaginal sonographic diagnosis of uterine cysts at different sites. Cyst aspiration was performed using interventional 2D ultrasonography, hysteroscopy and/or laparoscopy. Follow-up was performed for a maximum of 1 year to assess relief of symptoms and the recurrence rate. The main outcome measures of the study are success of aspiration tool, relief of symptoms, and persistence or recurrence rates. Cervical and corporeal uterine cysts were diagnosed in 19 and eight cases respectively. The mean size of the cervical cyst was $2.9 \pm 1.21$ $(1.8-3.7) \mathrm{cm}$, while that of the uterine cysts was $4.8 \pm 1.89$ $(3.4-6.1) \mathrm{cm}$. Improved health-related quality of life in the form of relief of deep dyspareunia and excessive vaginal discharge were reported in eight of $12(66.6 \%)$ and 11/14 $(78.5 \%)$ cases, respectively. Aspiration of symptomatic uterine cysts is technically feasible and achieves acceptable results. Uterine cysts may not be ignored as a potential cause of gynecologic symptoms, however, their precise pathogenesis and related co morbidities including impact on fertility should be substantiated by an adequately powered prospective randomized controlled study.
\end{abstract}

Keywords Uterine cysts · Ultrasonography · Laparoscopy · Hysteroscopy

A. M. Darwish $(\bowtie) \cdot$ K. M. Zahran • M. A. Bedaiwi •

M. S. Zakherah

Department of Obstetrics \& Gynecology,

Woman's Health University Hospital,

71111 Assiut P.O. Box: (1) Assiut, Egypt

e-mail: atef_darwish@yahoo.com

\section{Background}

Intrauterine adhesions, submucous myoma, adenomyosis uterii or sometimes uterine septa are the most frequently diagnosed uterine abnormalities in gynecologic practice. Most of these abnormalities can be easily diagnosed utilizing conventional 2D transvaginal ultrasonography with or without saline infusion [1]. On the other hand, uterine cysts are rarely seen with no reported incidence in medical literature and usually accidentally detected by ultrasonography. Commonly, they are cervical due to retention of Nabothian cysts. However, they may be cystic degeneration of uterine leiomyoma, cystic adenomyosis (adenomyotic cysts), congenital uterine cysts such as mesonephric and paramesonephric cysts, hydrosalpinx densely adherent to the myometrium, and echinococcal cysts [2]. Various kinds of congenital cysts cannot be differentiated from cystic adenomatoid tumors [3] which are relatively rare benign neoplasms that usually arise in the genital tract [4-7]. Intramyometrial abscess [8] or hydatid cyst [9] are other rare possibilities. Cervical cysts may be cysts of embryonic tissues, endometriotic, or endocervicotic cysts [10]. So far, there are no published studies on the imaging of uterine cysts, their impact on health-related quality of life or the optimal line of management of such cysts. This study aims to estimate the feasibility and efficacy of a minimally accessintegrated protocol for aspiration or drainage of symptomatizing sonographically diagnosed uterine cysts.

\section{Patients and methods}

This study was conducted at the Gynecologic outpatient clinic of the Woman's Health University Center, Assiut University, Assiut, Egypt between April 2001 and August 2008 and comprised 27 women with different gynecologic 
complaints and a transvaginal sonographic diagnosis of uterine cysts more than $2 \mathrm{~cm}$ in diameter at different sites.

Patients were counseled about participating in the study. A written informed consent was taken. Patients had the right to refuse to participate and/or withdraw from the study at any time without being denied their regular full clinical care. Personal information as well as data collected was subjected to confidentiality. The Institutional Review Board of the Assiut Faculty of Medicine approved this study.

Clinical work-up of the patients included entry history taking and thorough examination with stress on patients' complaints including type and duration of pelvic pain, coital-related pain, or abnormal vaginal discharge or backache. Infertile women were clearly informed that this study is not designed to help fertility. Patients with a clear potential cause of their complaints were excluded from this study, e.g., pelvic endometriosis, adenomyosis uteri, ovarian cysts, uterine fibroids, adnexal masses, pelvic inflammatory disease, or genital prolapsed. With the aid of transvaginal ultrasonography (TVS), uterine cysts appeared as a well defined hypoechogenic area not associated with uterine myomata or adenomyosis uterii. Uterine cysts more than $2 \mathrm{~cm}$ in diameter were included in this study. The relationship of the uterine cyst to the endometrial cavity or the cervical canal was meticulously defined. Comment on its size, fluid content, and borders were also reported. In case of suspicion, saline infusion sonohysterography (SIS) was performed in the same manner as previously described [1]. The couple was counseled for interventional 2D transvaginal sonographic aspiration of the cyst aiming at relief of the symptoms. Patients with corporeal cysts gave a written consent for laparoscopy in case of failed sonographic aspiration in the same operative setting. All procedures were done immediately postmenstrual. Under general anesthesia, TVS was performed. Sonographically guided aspiration of the cyst was done utilizing a 16-gauge aspiration needle principally designed for egg retrieval for assisted reproductive technique but used under higher pressure of the ordinary operating room suction unit at maximal power ( -0.8 to -1 bar) since the cyst content is usually different from the watery follicular fluid. The

Table 1 Basic data of the studied patients

\begin{tabular}{ll}
\hline Item & Result \\
\hline Age [years] [means \pm SD] (range) & $23.16 \pm 1.19(18-31)$ \\
Parity & $0.5 \pm 3.0(0-2)$ \\
BMI (kg/m2) (range) & $22.1 \pm 3.0(15-30)$ \\
Menstrual disturbances (oligo & $4(14.8 \%)$ \\
$\quad$ or hypomenorrhea) & $12(44.4 \%)$ \\
Deep dyspareunia & $14(51.8 \%)$ \\
\hline
\end{tabular}

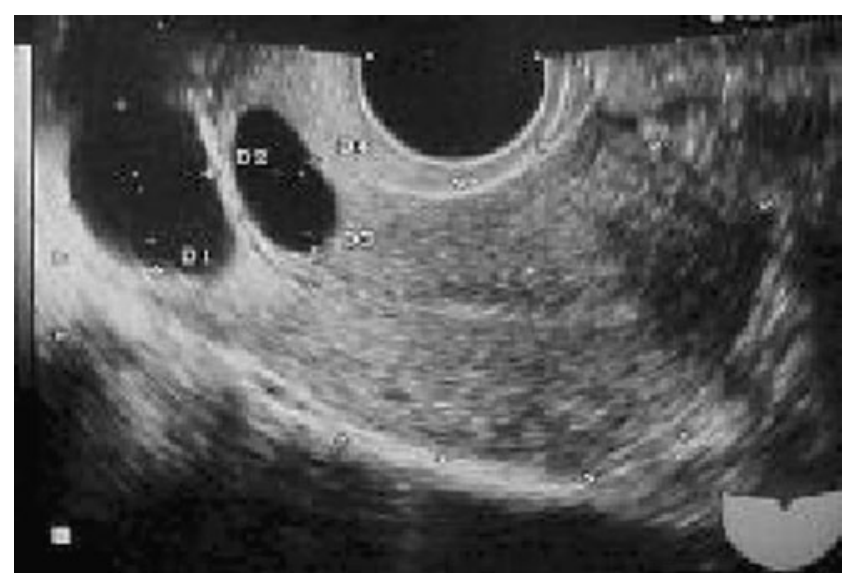

Fig. 1 Intracervical retention cysts compressing cervical canal

needle was loaded into an adaptor fitted to the vaginal probe. The procedure was considered completed once the cyst is seen entirely collapsed. The fluid content was subjected to cytologic examination after proper fixation with $95 \%$ alcohol. Failed access or aspiration of the viscid thick fluid content of corporeal cysts despite increased pressure of the suction unit were indication for operative laparoscopy in the same setting guided by the preoperative sonographic localization. Using a fine monopolar microneedle, a small incision was made over the cyst followed by drainage of its contents. The edges of the incision were coagulated with spray coagulation. The cyst content was aspirated for cytologic examination. On the other hand, if a part of the corporeal or cervical cyst is seen bulging into the endometrial or endocervical cavity respectively, a decision of hysteroscopic drainage was made. If an endocervical cyst was diagnosed, a sharp microscissors loaded into the side channel of the 7-mm operative hysteroscopy was used to puncture the cyst. If not accessible, a hook electrode of

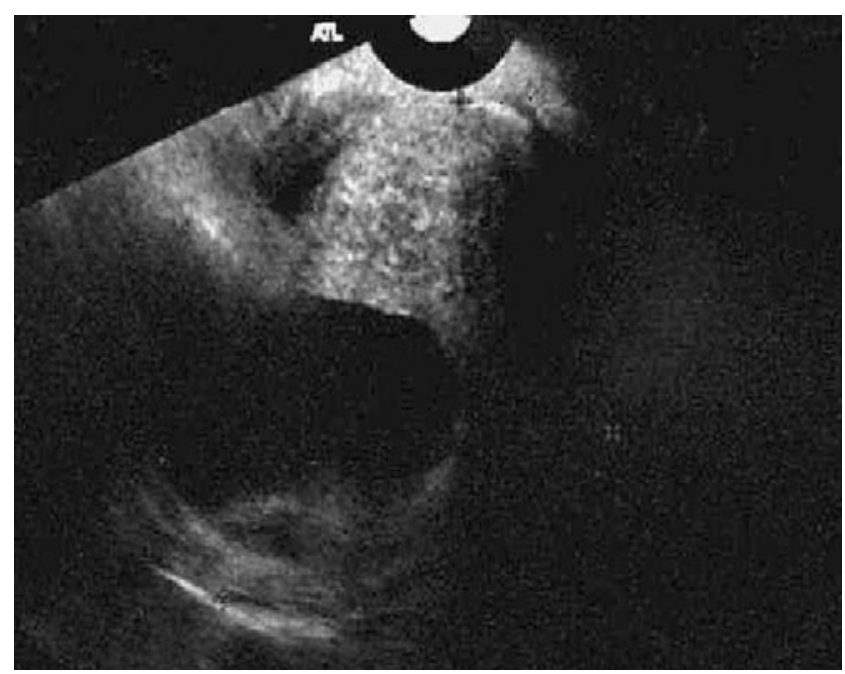

Fig. 2 A fundal corporeal cyst diagnosed by transvaginal ultrasonography 
Table 2 Sonographic findings

\begin{tabular}{lccc}
\hline & Cervical cysts 19 cases & Corporeal cysts eight cases & Significance $p$ value \\
\hline Cyst size $(\mathrm{cm})($ mean $\pm \mathrm{SD})$ & $2.9 \pm 1.212 .0-3.7$ & $4.8 \pm 1.89(3.4-6.1)$ & $\mathrm{NS}$ \\
Cyst near to the cavity & $11(57.8 \%)$ & $1(12.5 \%)$ & 0.03 \\
accessibility & $16(48.2 \%)$ & $5(62.5 \%)$ & NS \\
Successful sonographic aspiration & $13(68.4 \%)$ & $5(62.5 \%)$ & NS \\
\hline
\end{tabular}

$N S$ non significant

monopolar resectoscope was utilized for puncture and drainage. The same instrument was used to drain any cyst bulging into the endometrial cavity. Transabdominal sonographic monitoring may be required in some difficult cases.

At the end of the operation in all cases, a broad spectrum antibiotic was prescribed and the patient was discharged few hours postoperatively. One month later, all patients were examined with TVS to comment on the site of the cyst and any evidence of recollection.

Follow-up of all cases was continued for 1 year to assess for the improvement of deep dyspareunia, abnormal vaginal discharge, or other symptoms. Data were collected and analyzed with SPSS version 11 (SPSS, Inc., Chicago, IL, USA) and expressed as mean \pm standard deviation (SD). Statistical methods were applied including descriptive statistics (frequency, percentage, mean, and SD) and tests of significance $\left(X^{2}\right.$ and Fisher exact tests for categorical variables and Student $t$ test and Mann-Whitney tests for continuous variables). A $p$ value $\leq 0.05$ was considered statistically significant.

\section{Findings}

This study comprised 27 women with sonographic diagnosis of uterine cysts. Their basic data are demonstrated in Table 1. Cervical uterine cysts (Fig. 1) were diagnosed in

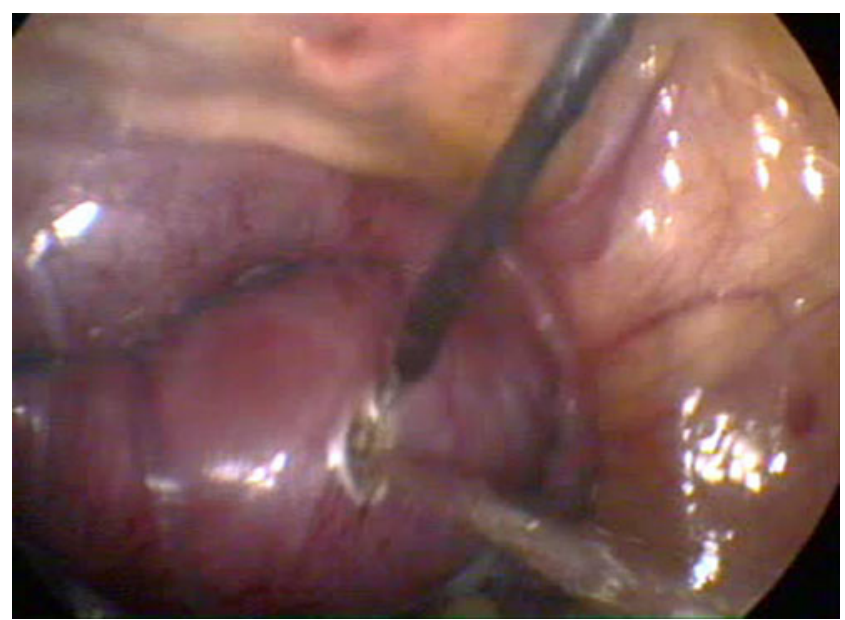

Fig. 3 Laparoscopic drainage of a fundal uterine cyst
19 cases, while corporeal cysts (Fig. 2) were diagnosed in eight cases. The mean size of the cervical cyst was $2.3 \pm$ $0.9 \mathrm{~cm}$, while that of the uterine cysts was $3.6 \pm 1.2 \mathrm{~cm}$. Details of transvaginal aspiration are demonstrated in Table 2. Failed sonographic access or aspiration of the corporeal cysts (two cases) was managed laparoscopically guided by the previous sonographic localization without complications (Fig. 3 and Table 3). Hysteroscopic approach was tried in 16 cases with cervical and one case with corporeal cysts (Table 3). Cytologic evaluation of the cyst aspirate revealed no malignant cells in all cases. On followup, patients reported subjective improvement of healthrelated quality of life in the form of relief of deep dyspareunia and abnormal vaginal discharge in eight of $12(66.6 \%)$ and $11 / 14(78.5 \%)$ cases, respectively; this difference was statistically significant (Table 4). We did not report cyst reformation in any case on follow-up for 1 year.

\section{Discussion}

Despite being not infrequently sonographically diagnosed, uterine cysts are frequently ignored as a potential cause of gynecologic symptoms. Others may miss the diagnosis of uterine cysts particularly cervical or fundal due to lack of orientation or awareness with uterine cyst(s). The most frequently seen uterine cysts are cervical cysts. A lot of gynecologists diagnose cervical cysts but consider them insignificant follicular cervicitis. Posterior cervical cysts can be easily mistaken with follicles in the Douglas pouch during sonographic folliculometry. Many case reports on rare types of uterine cysts were published [11]. Cystic adenomyosis is a rare form of adenomyosis of the uterine myometrium that has been described in adolescents and

Table 3 Results of aspiration

\begin{tabular}{lccccc}
\hline & \multicolumn{2}{c}{ Cervical cysts 19 cases } & & \multicolumn{2}{c}{ Corporeal cysts eight cases } \\
\cline { 2 - 3 } \cline { 5 - 6 } & Successful & Failed & & Successful & Failed \\
\hline Ultrasonography & 8 & 3 & & 4 & 2 \\
Laparoscopy & 0 & 0 & & 3 & 1 \\
Hysteroscopy & 11 & 5 & & 1 & 0 \\
\hline
\end{tabular}


Table 4 Persistence of the symptoms after 1 year of follow-up in the studied cases (27 cases)

\begin{tabular}{|c|c|c|c|}
\hline & $\begin{array}{l}\text { Before } \\
\text { procedures }\end{array}$ & $\begin{array}{l}\text { After } \\
\text { procedures }\end{array}$ & $\begin{array}{l}\text { Significance } \\
p \text { value }^{\mathrm{a}}\end{array}$ \\
\hline dyspareunia & 12 & 4 & $0.017^{\mathrm{a}}$ \\
\hline abnormal vaginal discharge & 14 & 3 & $0.001^{\mathrm{a}}$ \\
\hline
\end{tabular}

${ }^{\text {a }}$ Statistically significant

adults [12]. There are some reports on primary echinococcal cysts of the uterus [13]. Commonly, non-fibroid swellings are erroneously diagnosed as adnexal enlargements and their true origin only becomes evident during surgery [14]. Others reported a case of florid cystic endosalpingiosis in the subserosa of the uterine fundus which was clinically considered to be an ovarian tumor [11]. In this study, all cases of uterine cysts were easily diagnosed utilizing conventional high resolution 2D ultrasonography with clear demarcation from adnexa masses. Moreover, the use of SIS in doubtful cases would help detect uterine cysts after proper delineation of the external uterine surface by excessive fluid in the pouch of Douglas. By this way, MRI requested for such an indication [11] can be omitted.

Some published human studies managed uterine cysts with hysterectomy $[11,14]$. One case report on a complex uterine cyst demonstrated successful videohysteroscopic drainage utilizing electrocoagulation via a polypectomy snare guided by transrectal massage [15]. To the best of our knowledge, this study is the first publication in English and
non-English literature that addresses the use transvaginal 2D sonographic aspiration of uterine cysts in humans. Over a 6-year period, only 29 cases were reported in one series [14]. Of these, eight corresponded to the diagnosis of a nonfibroid uterine cystic enlargement, and 21 to that of a fibroid with cystic degeneration. Histologic diagnosis showed a cystic adenomyoma in three, a congenital cyst in three, and a blind rudimentary uterine horn in two cases, respectively. In this study, just cyst aspiration was performed since these rare cysts are usually benign in nature. Moreover, cytologic examination of the aspirate in all cases in this study revealed no evidence of malignant cells. Nevertheless, cyst aspiration is not an optimal approach. Ideally, the cyst wall should be peeled off to ensure non-reformation of the cyst. Since some patients in this study were infertile, a minimally invasive procedure has been chosen and approved by the ethics committee. Moreover, many of these cysts were inaccessible for surgical removal particularly cervical retention cysts. Previously, we utilized a monopolar needle for blind puncture of these cervical cysts. However, we completely stopped this procedure due to reported cases of iatrogenic cervical atresia. Later on, we tried hysteroscopic opening of intramural cysts but unfortunately all these trials failed due to non-visualization of the cyst unless it protrudes into the cervical canal. Even after resectoscopic opening of the protruding cysts, cyst wall extraction was impossible and at the end it became just fenestration technique. In this study, hysteroscopic approach was restricted to any cyst bulging into the endocervical canal or the endometrial cavity where we achieved excellent results (11/16 and one of one

Fig. 4 Management plan of uterine cysts

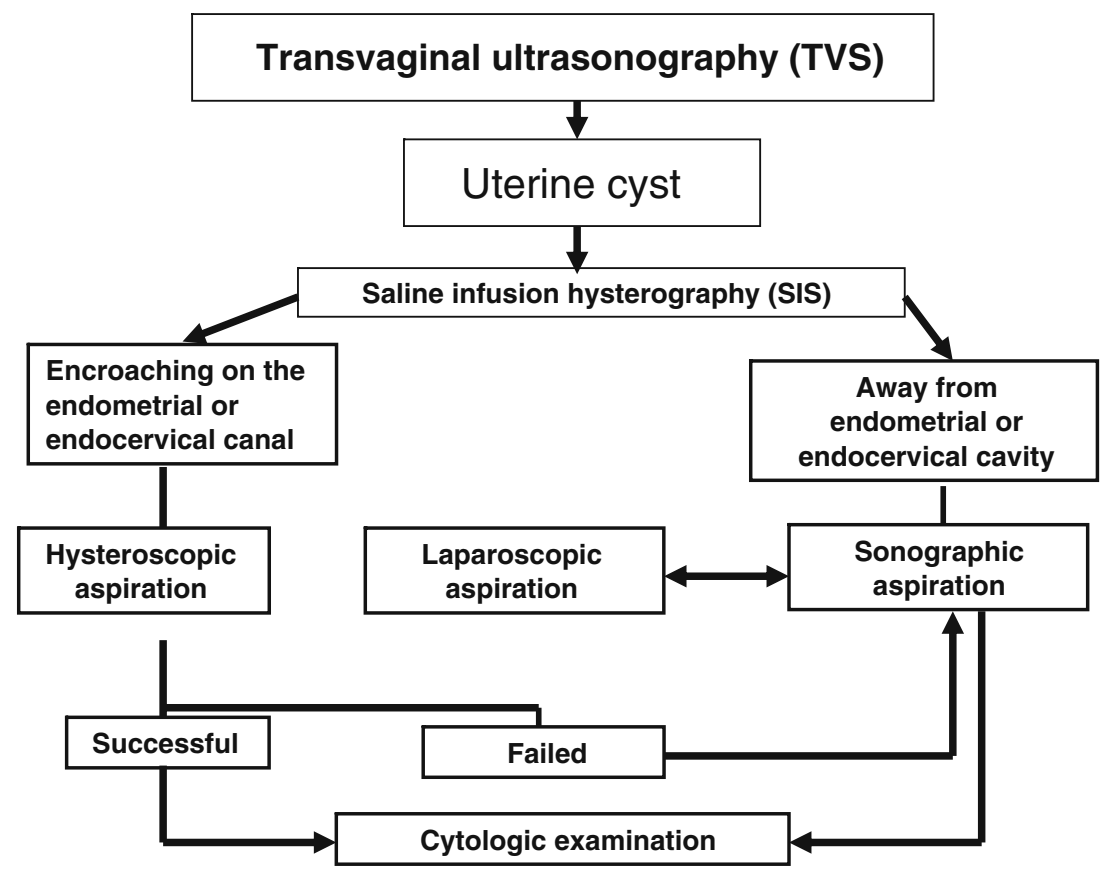


cervical and corporeal cysts, respectively). This study opens the door for a new era of hysteroscopic access to the myometrial lesions. Nevertheless, unlike hysteroscopy, sonographic approach is easier, non-invasive, available, and effective. This study addresses a laparoscopic back-up approach for treating uterine cysts. It is a simple and effective method of drainage. Moreover, those infertile women would get much more benefit of laparoscopic evaluation of their pelvis with concomitant management of any associated cause of infertility. Furthermore, we did not report cyst reformation in any case on follow-up for 1 year. This can be explained by the nature of these cysts which seems to be of embryonic origin lined by poorly developed glands in most of cases.

The impact of these cysts on fertility as well as the patient's health-related quality of life is unknown. Proposed mechanisms of impaired fertility secondary to uterine cysts would include mechanical compression of the endocervical canal or the endometrial cavity, dysrhythmic uterine contractions that interfere with proper implantation, or chronic irritation and infection. Nevertheless, we excluded fertility and pregnancy rates following cyst aspiration or drainage among infertile cases to avoid dissociation of the message of this work.

\section{The limitations of the study}

Failure to obtain cyst wall for histopathologic examination to determine the cyst nature is a clear limitation of this study. Nevertheless, we omitted this step since most of the scanty publications on uterine cysts demonstrated benign nature. Moreover, trials of cyst wall extraction or even coagulation of the whole cyst would invite adhesions for those women with unexplained infertility. Failed aspiration in some cases due to viscid nature of the fluid is another real limitation. This problem can be overcome by utilizing a larger needle caliber in the subsequent trials. Failed hysteroscopic aspiration would be minimized if concomitant sonographic guidance is used. Limited number of cases is another clear limitation of this study. Further similar studies with sufficient sample size are needed. The material of this study is quite mixed with respect to site and management options.

It is concluded that uterine cyst aspiration or drainage following an integrated protocol utilizing ultrasonography, hysteroscopy and/or laparoscopy (Fig. 4) is feasible and achieves acceptable results. While it is good to point out that uterine cysts should not be ignored, the claim that their impact on fertility and health-related quality of life is not substantiated. A randomized prospective study of large sample size would be required.

Acknowledgments We would like to thank residents and staff members of the ultrasonographic and endoscopic units of the Woman's Health University Center, Assiut University, Assiut, Egypt for their generous effort during the long study period.

Financial support None.

Declaration of interest The authors report no conflicts of interest. The authors alone are responsible for the content and writing of the paper.

\section{References}

1. Darwish AM, Youssef AA (1999) Screening sonohysterography in infertility. Gynecol Obstet Investig 48(1):43-47

2. Kataoka ML, Togashi K, Konishi I (1998) MRI of adenomyotic cyst of the uterus. J Comput Assist Tomogr 22:555-559

3. Kim JY, Jung KJ, Sung NK, Chung DS, Kim OD, Park S (2002) Cystic adenomatoid tumor of the uterus. American Roentgen Ray Society Journal 179:1068-1070

4. Bisset DL, Morris JA, Fox H (1988) Giant cystic adenomatoid tumour (mesothelioma) of the uterus. Histopathology 12:555-558

5. Mitsumori A, Morimoto $M$, Matsubara $S$, Yamamoto $M$, Akamatsu N, Hiraki Y (2000) MR appearance of adenomatoid tumor of the uterus. J Comput Assist Tomogr 24:610-613

6. Livingston EG, Guis MS, Pearl ML, Stern JL, Brescia RJ (1992) Diffuse adenomatoid tumor of the uterus with a serosal papillary cystic component. Int J Gynecol Pathol 11:288-292

7. Rosa GD, Boscaino A, Terracciano LM, Giordano G (1992) Giant adenomatoid tumors of the uterus. Int J Gynecol Pathol 11:156-16

8. Erguvan R, Meydanli MM, Alkan A, Gokce MN, Kafkasli A (2003) Abscess in adenomyosis mimicking a malignancy in a 54year-old woman. Infect Dis Obstet Gynecol 11:59-64

9. Kavak BZ, Gökaslan H, Küllü S (2002) Hydatid cyst of the uterus. Infect Dis Obstet Gynecol 10(2):67-70

10. Tindal VR (1987) Jeffcoate's principles of gynaecology, 5th edn. Butterworths, London, p 396

11. Sang Hwa Shim, Han-Seong Kim, Mee Joo, Sun Hee Chang, Ji Eun Kwak (2008) Florid cystic endosalpingiosis of the uterus-a case report. The Korean Journal of Pathology 42:189-191

12. Ho ML, Raptis C, Hulett R, McAlister WH, Moran K, Bhalla S (2008) Adenomyotic cyst of the uterus in an adolescent. Pediatr Radiol 38(11):1239-1242

13. Langley GF. Primary echinococcal cyst of the uterus British Journal of Surgery Jan1943;30,119,278-280 Online: Dec 62005.

14. Protopapas A, Milingos S, Markaki S, Loutradis D, Haidopoulos D, Sotiropoulou M, Antsaklis A (2008) Cystic uterine tumors. Gynecol Obstet Investig 65:275-280

15. Rambags BP, Stout TA (2005) Transcervical endoscope-guided emptying of a transmural uterine cyst in a mare. Vet Rec 156:679682 